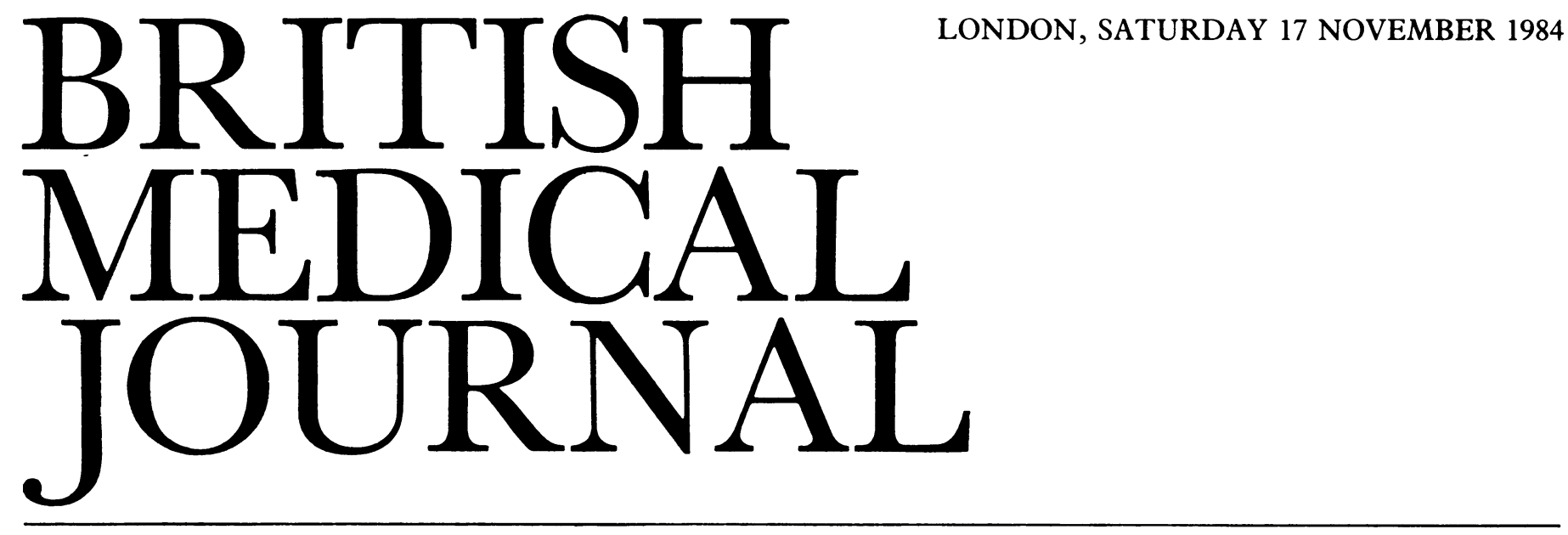

\title{
Tuberculosis in hospital doctors
}

With the decline in the prevalence of tuberculosis over the past 30 years there has grown a disturbing air of complacency. A leading article ${ }^{1}$ in the $B M \mathcal{F}$ in 1973 drew attention to the growing misconception among doctors that tuberculosis had become a rare disease. ${ }^{2}$ Though only a few cases of tuberculosis may be seen in some regions, in others the incidence of the disease is comparatively high. Susceptible groups such as homeless alcoholics and Asian immigrants present their own particular problems. ${ }^{3-5}$ When, however, a low index of clinical suspicion is compounded by failure to recognise an atypical presentation in a patient admitted to a general medical ward the diagnosis may not be made until necropsy..$^{6.9}$

The incidence of tuberculosis in hospital medical staff in Britain has not been documented, so no numbers can be put on the risk of tuberculosis among doctors. Screening programmes for tuberculosis have been carried out on hospital employees including nurses and medical staff in Canada, the United States, and Britain. ${ }^{10-14}$ Individual cases of tuberculosis in hospital doctors are reported from time to time. ${ }^{15}$ Any individual with undiagnosed respiratory tuberculosis presents a hazard, whether he is a hospital patient or employee. Infection may spread from either source" - as was shown in Ontario, where a woman patient who died three days after admission for "bronchopneumonia" and in whom acid fast bacilli were found in the sputum the day before death had infected another patient and seven members of the hospital staff. In another example a resident doctor in a paediatric department was diagnosed as having sputum positive pulmonary tuberculosis. Less than half of his colleagues attended for routine chest radiographs - though four child patients had been infected and three had evidence of pulmonary tuberculosis. ${ }^{15}$

The recent publication of a report from Leeds of six cases of active tuberculosis in doctors invites comment. ${ }^{16}$ Memoranda on the protection of hospital staff against tuberculosis issued by the Ministry of Health have been updated by the Department of Health and Social Security (DHSS). ${ }^{17} 18$ In October 1983 the Joint Tuberculosis Committee of the British Thoracic Society published its code of practice ${ }^{19}$ on the control and prevention of tuberculosis, incorporating advice given in the DHSS health circular $\mathrm{HC}(78) 3 .{ }^{18}$ This is an important and welcome document which has been compiled with great care. The code recommends that staff at normal risk do not need regular chest radiographs, but one taken at the termination of employment may be advisable in view of current industrial injuries legislation. ${ }^{20}$ Joint correspondents to the code of practice warned that staff in "minimal risk" wards may also be exposed to new cases of tuberculosis and pointed out that there is no screening programme for professional foreign visitors attached to NHS hospitals. ${ }^{21}$

To protect susceptible patients all staff working in obstetric and children's departments should provide evidence of a satisfactory chest radiograph taken within the previous six months - as recommended in the DHSS health circular ${ }^{18}$ rather than within the previous 12 months, as recommended in the code of practice. In the context of pre-employment examination of NHS staff at normal and higher risk the code makes no reference to previous BCG vaccination. This is unfortunate, particularly since vaccination is advised for all those who have either negative or grade 1 reactions to the multipuncture (Heaf) tuberculin test. The tuberculin reaction after BCG vaccination is generally weak (Heaf or tine grades 1 or 2 ), so that a grade 1 reaction in a previously vaccinated person is reasonable evidence of adequate protection against tuberculosis. ${ }^{22}$ In the absence of previous BCG vaccination a grade 1 reaction is strong evidence of previous mycobacterial infection, which provides adequate protection. ${ }^{22}$ In such cases BCG vaccination may safely be withheld -indeed if it is given it may produce large scars. On the other hand, if in a previously vaccinated person the Heaf test gives a negative result, a Mantoux test (100 IU) should be performed; if this too is negative repeat BCG vaccination would be justified.

Tuberculin testing is important for hospital staff. ${ }^{23}$ Large surveys on nurses and other young adults have shown that the risk of developing tuberculous lesions is greatest in the 12 to 18 months after tuberculin conversion. ${ }^{1324}$ In the Leeds study five of the six doctors with active tuberculosis were junior hospital doctors, and three were in close contact with children. ${ }^{16}$ Only two of the doctors, one of whom worked with children, had received pre-employment screening. Both had had strongly positive Heaf test results; these were attributed to previous BCG vaccination so that they were not followed up. The presence of a strongly positive tuberculin reaction should in fact have aroused suspicion of probable 
superinfection by Mycobacterium tuberculosis. A repeat chest radiograph taken three months later would have been a wise precaution. In the event both doctors were discovered to have active disease six months later.

Compared with other hospital workers, doctors are the least compliant in caring for themselves; those at greatest risk are pathology staff who handle infected specimens. ${ }^{25}$ They do not - though some would like to believe it - possess divine immunity from all ills, and it is in their own interest as well as that of their colleagues and patients to present for preventive examination. A well coordinated occupational health service in the NHS is overdue. ${ }^{26}$

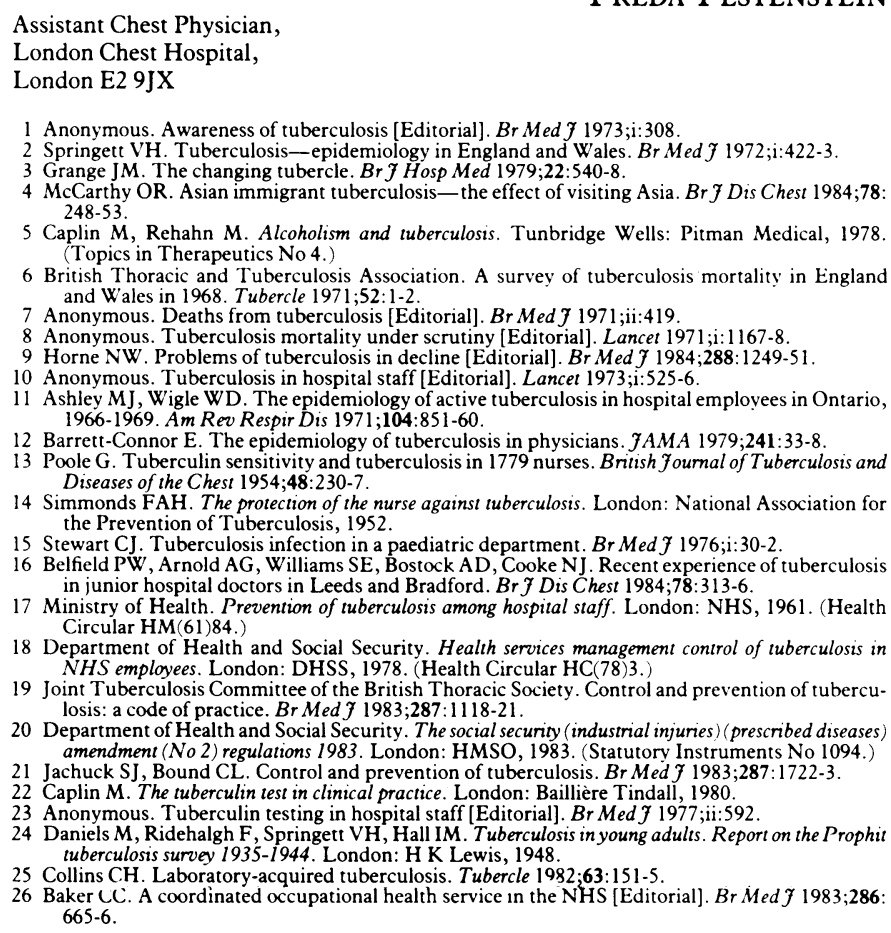

\section{Lymphocytes are rhythmic: is this important?}

Immunology is now moving into a new era as new techniques make many established measurements easier and as new, often more complex, analyses show that earlier classifications were too simple. For example, new methods have allowed us to classify lymphocyte populations and to describe subsets of these with immunofluorescent flow cytometry.

Thirty years ago endocrinologists moved into their new era with new methods of measuring hormones and of discovering new hormones and prohormones. As their techniques became simpler and better they were able to make repetitive and more precise measurements. One of the discoveries that followed was that the secretion of hormones is episodic and often rhythmic. Some patterns were no surprise; the hormones concerned in the menstrual rhythms would be expected to be secreted on a cyclical basis. When growth hormone was first measured, however, it was some surprise to find that the pituitary discharges this hormone in episodic bursts, very few during the day and most in the period around waking. (Dawn is therefore the time to identify a growth hormone deficiency.)
Serum cortisone measurements in blood were also found to vary throughout the 24 hours, and initial studies based on six to eight points suggested an almost sinusoidal pattern. In fact, the initial descriptions of the adrenal rhythm turned out to be an oversimplification. Very frequent sampling showed that the rhythm results from episodic surges of secretion of variable frequency-with the surges again being most frequent before waking. ${ }^{1}$

At first these new facts caused difficulties for clinicians concerned with endocrinology, but as they became accepted they were slowly incorporated into strategies of investigation and management. Immunologists are now faced with the evidence that there are similar time dependent variations in the immune system and must respond to them.

Physicians concerned with immunology must now follow the example of their colleagues in endocrinology and accept as a fact that the immune system is rhythmic. Firstly, we need to decide at what time of day samples used for measurement are to be taken. In some conditions samples may best be taken in the morning, for others in the evening, and in some repetitive sampling may be appropriate. The recognition and definition of any rhythms or episodic variations present should now be considered an essential part of the investigation of any newly identified body constituent, and this approach is essential if the best use is to be made of new methods of assay. Indeed, there may be some circulating materials that come out only at night but are of importance and are still to be discovered because of a failure to consider this possibility.

Studies on circadian changes in $\mathrm{T}$ lymphocyte subsets were the main topics of two papers in the $B M \mathcal{F}$ and also in other papers published elsewhere during 1983.2.5 Some of these publications and others on immunology and immune responses are discussed below, already having been reviewed by us in other journals. ${ }^{677}$ Some disease processes are also episodic, and as the immune or inflammatory responses that are associated with them are rhythmic there may well be a relation. ${ }^{89}$ The episodic "rhythmic" changes seen in biology that may influence disease range in frequency with evidence of rhythms that are ultradian (shorter than 24 hours), circadian (around 24 hours), and infradian (greater than 24 hours-for example, around seven days (circaseptan), around 28 days and yearly (circannual)).

The study of rhythms in the immune system that may be relevant to the causes of illness, or to the body response to an illness, has been neglected by immunologists and by physicians despite elegant and well documented studies of rhythms in the number of circulating white blood cells and lymphocytes, ${ }^{10} 11$ the responses of lymphocytes in vitro, ${ }^{12-14}$ and the immune responses to which they contribute. ${ }^{15} 16$ There are differences in the anti-inflammatory and immunosuppressant effects of a drug when the same dose is given at different times. ${ }^{17-19}$ We have suggested, supported by evidence which is circumstantial rather than conclusive, that events of clinical importance which are episodic in their manifestations may be the result of the immune system (or the associated inflammatory responses) being rhythmic. Two examples are the early morning exacerbations in rheumatoid arthritis, ${ }^{8}$ and the tendency for the onset of rejection of a renal allograft to take place at night, ${ }^{9}$ and with recurrences around every seven days. ${ }^{20}$ The absence of descriptions of repetitive clinical patterns in other clinical settings where the immune system is concerned might simply be due to the appropriate studies not having been done. Negative studies tend to gather dust in an investigator's file, and clinical studies showing the absence of rhythms may exist but have not been published. If 